open surgery. However, the results must be interpreted with caution given the potential risk of bias derived from the relatively small sample size and the single-centre, retrospective nature of the study.

Conclusion In the present study, we did not find statistically significant differences between LRH and ORH for the treatment of early-stage cervical cancer in terms of disease-free survival and overall survival.

Disclosures Nothing to disclosure.

\section{CERVICAL ONCOLOGICAL CYTOLOGIES'S (PAP TEST) EVALUATION OF THE RIVERINE WOMEN FROM RIVERS TAPAJÓS AND CUPARI (PARÁ - BRAZIL)}

Maria Vitoria Lima Ferrero, Rafaela Estefano Uriza, Aline Silva Moreira Dos Santos, Letícia Bertaglia, José Anibale Rodrigues Junior, Jhenifer Nataly Moura França, Fabiana Moreira Passos Succi, Icléia Siqueira Barreto, Katia Piton Serra. São Leopoldo Mandic

\subsection{6/ijgc-2020-ESG0.12}

Introduction/Background Cervical cancer is the most prevalent in Brazil North Region. There are few studies about cervical injuries in the riverine women. The knowledge of these characteristics could improve health assistance to these women, contributing to health policies that benefit this population. Objective: This paper aims to evaluate the cervical oncological cytologies of the riverine women from rivers Tapajós and Cupari (Pará - Brazil).

Methodology A partnership between SLMandic Medical School and the local Cities of Santarém, Aveiro and Belterra (State of Pará, Brazil) Government was established trhough 'Barco da Saúde' (Health Boat) project. The Counties have provided a Hospital Boat named 'Abaré'. SLMandic has provided a team made up by professors and medical students. Services in pediatrics, gynecology and obstetrics, dermatology, ophthalmology and odontology were provided. In 2019, the activities were held between July 27nd and August 5th, 206 gynecological and obstetric appointments were made and 133 cervical oncological cytologies were collected. Epidemiological data were collected from anamnesis form designed specifically for the expedition. The reading of the cytology slides was performed and de injuries classified among high grade lesions (HSIL), low grade lesions (LSIL), scaly lesions of undetermined significance (ASC-US), and glandular lesions of undetermined significance (ASCH). Pathological agents found have also been described. The samples were classified between satisfactory and unsatisfactory. Finally, statistical analysis of data was done.

Results Out of 206 gynecological and obstetric appointments, $132(64.1 \%)$ cervical cytologies were collected. Among these patients, $100(75.75 \%)$ were screening age (25-65 years old). Median of patients age was 34.4 years old. About 128 (97\%) samples were satisfactory for analysis. Cervical cancer screening was the objective of the exam in 101 (76.5\%) of the cases. Related to associated microorganisms, Lactobacillus sp. were present in 68 (51.5\%), Gardnerella vaginallis in 13 (9.8\%), Candida sp. in $8(6 \%)$ Trichomonas vaginallis in 2 $(1,5 \%)$, in addition to others less prevalent. Inflammatory cells were present in $126(95.5 \%)$ of the cases. The main diagnosis was benign cellular changes $(122-92.5 \%)$ and atypical squamous cells of undetermined significance, ASC-US, 6 (4.5\%). Were performed 7 (5.3\%) biopsies, being 3 (42.9\%) cervical polyps and $4(57.1 \%)$ nonspecific chronic cervicitis.
Conclusion Despite difficulties to access health care, health indicators were better than expected. There was good cytological coverage and no high-grade lesions were found.

Disclosures Authors declare no conflict of interest.

\section{A CLINICAL STUDY ON THE APPLICATION OF 3D- PRINTING MINIMALLY INVASIVE-GUIDED TEMPLATE IN BRACHYTHERAPY OF PATIENTS WITH LOCALLY ADVANCED CERVICAL CANCER}

Xiangkun Yuan, Yongxia Zhang, Junjun Miao. Hebei Province Cangzhou Hospital of Integrated Traditional and Western Medicine

\subsection{6/ijgc-2020-ESG0.13}

Introduction/Background To explore the clinical application of three-dimensional (3D)-printing minimally invasive-guided template in brachytherapy of patients with locally advanced cervical cancer.

Methodology From May 2016 to December 2018, 59 patients (age, 23-78 years old; median age, 57 years old) with locally advanced cervical cancer. All patients were treated with radical radiotherapy, in which external irradiation was performed, and 3D conformal and intensitymodulated radiotherapy was carried out with a radiation dose of $45 \mathrm{~Gy}$ in 25 fractions of 1.8 Gy per day. The included patients were randomly divided into 2 groups according to random number table method. In the template group, 29 patients assisted by $3 \mathrm{D}$-printing templates to place intrauterine tubes and implant for insertion of needles. In the free implantation group, 30 patients were assisted with freehand implanted intrauterine tubes and implant needles. All patients underwent Computed Tomography (CT) to adjust the position and depth of the insertion needle, and the final CT image was transmitted to the Oncentra brachytherapy planning system, to outline the target area and organs at risk, make treatment plans, and perform treatment.

Results A total of 283 times of combination of intra-luminal and interstitial insertion radiotherapy were undertaken, and a total of 283 times of post-loading radiotherapy plan were formulated, including 141 times in template group and 142 times in free insertion radiotherapy. Complete Response (CR) rate in the template group $(24 / 28 ; 85.71 \%)$ was slightly higher than that in the free transplantation group (22/28; $78.57 \%)$. There was no significant difference in short-term efficacy between the two groups $(z=-0.692, P>0.05)$. Importantly, D90 (90\% of the target volume) of High-Risk Clinical Target Volume (HR-CTV) and Intermediate-Risk Clinical Target Volume (IR-CTV) in the template group were significantly higher than those in the free implantation group $(\mathrm{t}=$ $3.42,2.13, \mathrm{P}<0.05)$. D2 $\mathrm{cm}^{3}$ of bladder, rectum and sigmoid colon was significantly reduced $(\mathrm{t}=-2.59,-4.22,-2.01$, $\mathrm{P}<0.05)$. Therefore, the incidence of grade 1,2 and 3 acute radiation proctitis in the template group was noticeably lower than that in the free transplantation group $(z=-2.112$, $\mathrm{P}<0.05)$. However, there was no significant difference in the incidence of acute radioactive cystitis between the template group and the free implantation group $(\mathrm{z}=-1.686, \mathrm{P}>$ 0.05).

Conclusion For large-block or eccentric cervical cancer, application of the 3D-printing minimally invasive-guided template in brachytherapy of patients with locally advanced cervical 
cancer can reflect its dose-based advantages, associating with a remarkable reduction of patients' adverse reactions and a satisfactory therapeutic effect.

Disclosures None.

\section{HIGH EXPRESSION OF NANOG AND CRY1 IS INVOLVED WITH TUMOR PROGRESSION AND POOR PROGNOSIS IN PATIENTS WITH CERVICAL CANCER}

${ }^{1}$ Gwan Hee Han, ${ }^{2}$ Hanbyoul Cho. ${ }^{1}$ Department of Obstetrics and Gynecology, Yonsei University, College of Medicine; Department of Obstetrics and Gynecology, Kyung Hee University Hospital at Gangdong; ${ }^{2}$ Department of Obstetrics and Gynecology, Yonsei Univeristy, College of Medicine

\subsection{6/ijgc-2020-ESGO.14}

Introduction/Background Nanog is a well-known transcription factor regulating an embryonic stem cell maintenance. Recently, many evidences have been accumulated that overexpression of Nanog is intimately involved in tumorigenesis. However, the role of Nanog in cervical cancer has not been elucidated yet. Thus, we investigated the expression and clinical significance of Nanog in cervical cancer.

Methodology To evaluate the expression level of NANOG and CRY1, the immunohistochemistry on 170 cervical cancers and 263 cervical intraepithelial neoplasia (CIN) samples and the clinicopathologic variables of cervical cancer patients were compared to evaluate the significance of Nanog and CRY1 in cervical cancer. Also, in vitro assessment was performed by using Nanog knock down cervical cancer cell lines.

Results Nanog and CRY1 expression was higher in cervical cancer tissues than in CIN tissues and normal epithelial tissues (both $\mathrm{p}<0.001$ ). Importantly, Nanog and CRY1 overexpression was associated with poor chemoradiation response $(\mathrm{p}<$ $0.035, \mathrm{p}<0.003$, respectively). Multivariate survival analysis revealed that overexpression of Nanog (hazard ratio $=0.016$; $95 \%$ confidence interval [CI]: $1.25-9.27), \mathrm{p}=0.016)$ as an independent prognostic factor for overall survival. Also, the combination of high Nanog and CRY1 expression showed the highest hazard ratio (5.87; 95\% CI: 2.18-15.82, p < 0.001) for overall survival. In vitro results also demonstrated the knockdown of Nanog was associated with increased cell viability $(\mathrm{p}<0.001)$, migration $(\mathrm{p}<0.001)$ and growth $(\mathrm{p}<$ 0.001 ) supporting the oncogenic role of Nanog in cervical cancer.

Conclusion This study showed that overexpression of Nanog could be a good biomarker for the prediction of chemoradiation response. The results of survival analysis suggest a strong association between Nanog as well as CRY1 expression and poor overall survival, indicative of the potential role of this combination as a prognostic marker in clinical assessment.

Disclosures To the best of our knowledge, the named authors have no conflict of interest, financial or otherwise.

\section{PERFORMANCE AND DIAGNOSTIC ACCURACY OF HUMAN PAPILLOMAVIRUS TESTING ON URINE AND SELF-COLLECTED VAGINAL SAMPLES IN A REFERRAL POPULATION}

Hyun-Woong Cho, Jae Kwan Lee, Jin Hwa Hong. Korea University Guro Hospital
Introduction/Background Human papillomavirus (HPV) is well established as the main cause of cervical cancer. Non-invasive self-collected urine and vaginal sampling have the potential advantage of increasing patient compliance with cervical cancer screening.

Methodology Women referred for colposcopy at Korea University Guro Hospital, following abnormal cytology, were included this study. A total of 314 paired urine, vaginal and cervical samples were collected. Primary endpoints were sensitivity for CIN2+/CIN3 + and specificity for <CIN2; secondary endpoints were the relative accuracy of hrHPV test results in vaginal and urine samples versus cervical samples.

Results For clinician-collected cervical samples, Sejong Realtime HR-S HPV test sensitivity for detecting and specificity from were similar to well-established test (Anyplex ${ }^{\text {TM }}$ II HPV 28) [sensitivity for CIN3+ $(\mathrm{n}=109) \quad 93.27 \% \quad(95 \%$ confidence interval (CI), 86.62-97.25); CIN2+ $(n=130) \quad 92.74 \% \quad(95 \%$ CI, 86.67-96.63); specificity for $<$ CIN2 31.82\% (95\% CI, 25.01-39.25)]. All the paired differences (cervical versus urine sampling, cervix versus vaginal sampling) in sensitivity were statistically significant. However, among women with ASCUS/ LSIL cytology, hrHPV sensitivity on vaginal samples was comparable to that of cervical samples for detection of CIN2+ and CIN3 + lesions. In addition, hrHPV sensitivity of Anyplex II HPV 28 assay on urine was comparable to that of cervical samples for detection of CIN3 + lesions $(p=0.07)$ in women with ASCUS/LSIL cytology.

Conclusion HPV tests using urine and vaginal samples were still inferior to clinician-collected cervical samples in terms of detecting CIN2/3. However, these results indicate that combination of cytology with reflex hrHPV test using vaginal and urine samples may offer a reliable strategy for discriminating women at greater risk of precancerous lesion, increasing compliance of patients.

Disclosures I have no conflict of interest to disclose.

\section{SUCCOR CONE: IS IT CERVICAL CONIZATION A PROTECTIVE MANEUVER}

${ }^{1}$ Enrique Chacón, ${ }^{2}$ Nabil Manzour, ${ }^{3}$ José Ángel Mínguez, ${ }^{3}$ Daniel Vázquez, ${ }^{3}$ Teresa Castellano, ${ }^{3}$ Luisa Sánchez Lorenzo, ${ }^{3}$ Diego Salas, ${ }^{4} J u a n$ Luis Alcazar, ${ }^{3}$ Antonio Gonzalez-Martin, ${ }^{3}$ Luis M Chiva. ${ }^{1}$ Clínica Universidad de Navarra; Obstetrics and Gynecology; ${ }^{2}$ Clínica Universidad de Navarra; Clínica Universidad de Navarra; Gynecology; ${ }^{3}$ Clínica Universidad de Navarra; ${ }^{4}$ Clínica Universidad de Navarra; Clinica Universidad de Navarra

\subsection{6/ijgc-2020-ESGO.16}

Introduction/Background After the publication of the LACC trial, current evidence has focused on looking for the different reasons that have led to the open approach presenting better results than minimally invasive surgery (MIS). To date, no studies have considered the possible protective effect of cervical conization (CC).

Methodology Objective: The main goal of this study was to compare disease-free survival (DFS) and overall survival (OS) at 4.5 years in patients with stage IB1 cervical cancer who underwent radical hysterectomy (2013-2014) after CC vs nonCC patients. The secondary goal was to compare DFS by subgroups (tumor size and surgical approach in patients who underwent CC and those who did not) in the Propensity Matching Score (PMS) database.

Methods: Taking from 1272 patients from the European database belonging to the SUCCOR study and after applying 